\title{
CARTESIAN CLOSED 2-CATEGORIES AND PERMUTATION EQUIVALENCE IN HIGHER-ORDER REWRITING
}

\author{
TOM HIRSCHOWITZ
}

CNRS, Université de Savoie

e-mail address: tom.hirschowitz@univ-savoie.fr

\begin{abstract}
We propose a semantics for permutation equivalence in higher-order rewriting. This semantics takes place in cartesian closed 2-categories, and is proved sound and complete.
\end{abstract}

\section{INTRODUCTION}

Cartesian closed categories provide semantics for equational theories with variable binding [12, 4]. On the other hand, 2-categories with finite products provide semantics for term rewriting [3]. The present paper shows that cartesian closed 2-categories provide semantics for term rewriting with variable binding, as embodied by Brugginks's generalisation [1] of permutation equivalence [16, Chapter 8] to higher-order rewriting [11, 19, 15, 17].

We first define cartesian closed 2-signatures, which generalise higher-order rewrite systems, and organise them into a category Sig. We then construct an adjunction

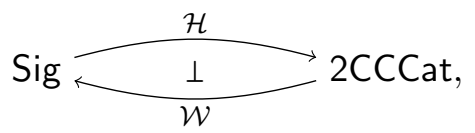

where 2CCCat is the category of small cartesian closed 2-categories. From a given cartesian closed 2-signature $S$, the functor $\mathcal{H}$ constructs a cartesian closed 2-category, whose 2-cells are Bruggink's proof terms modulo permutation equivalence, which we prove is the free cartesian closed 2-category generated by $S$.

We review a number of examples and non-examples, and sketch an extension to deal with the latter.

2012 ACM CCS: [Theory of computation]: Semantics and reasoning-Program semanticsCategorical semantics / Denotational semantics / Operational semantics.

Key words and phrases: Cartesian closed 2-categories, lambda calculus, higher-order rewriting, combinatory reduction systems, categorical semantics.

Partially funded by the French ANR projets blancs PiCoq ANR-10-BLAN-0305 and Récré ANR-11-BS020010 . 
Related work. Our cartesian closed 2-signatures may be seen as a 2-dimensional refinement of cartesian closed sketches [18, 5, 10]. Bruggink's calculus of permutation equivalence is close in spirit to Hilken's 2-categorical semantics of the simply-typed $\lambda$-calculus [8], but technically different and generalised to arbitrary higher-order rewrite systems. Capriotti [2] proposes a semantics of so-called flat permutation equivalence in sesquicategories. More related work is discussed in Section 4.2 .

\section{Cartesian Closed signatures and Categories}

We start by recalling the well-known adjunction [12, 4] between what we here call (cartesian closed) 1-signatures and cartesian closed categories.

For any set $X$, define types over $X$ by the grammar:

$$
A, B, \ldots \in \mathcal{L}_{0}(X) \quad::=x|1| A \times B \mid B^{A},
$$

with $x \in X$.

Proposition 2.1. $\mathcal{L}_{0}$ defines a monad on Set.

Let the set of sequents over a set $X$ be $\mathcal{S}_{0}(X)=\mathcal{L}_{0}(X)^{*} \times \mathcal{L}_{0}(X)$, i.e., sequents are pairs of a list of types and a type. The assignment $X \mapsto \mathcal{S}_{0}(X)$ extends to an endofunctor on Set.

Definition 2.2. A 1-signature consists of a set $X_{0}$ of sorts, and an $\mathcal{S}_{0}\left(X_{0}\right)$-indexed set $X_{1}$ of operations, or equivalently a map $X_{1} \rightarrow \mathcal{S}_{0}\left(X_{0}\right)$.

A morphism of 1-signatures $\left(X_{0}, X_{1}\right) \rightarrow\left(Y_{0}, Y_{1}\right)$ is a pair $\left(f_{0}, f_{1}\right)$ where $f_{i}: X_{i} \rightarrow Y_{i}$ such that

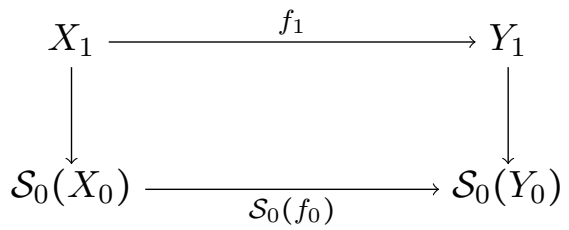

commutes. Morphisms compose in the obvious way, and we have:

Proposition 2.3. Composition of morphisms is associative and unital, and hence 1-signatures and their morphisms form a category $\mathrm{Sig}_{1}$.

There is a well-known adjunction

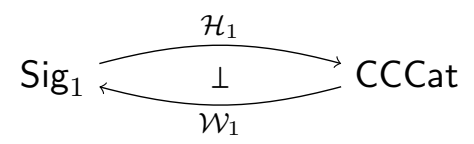

between 1-signatures and the category CCCat of small cartesian closed categories (with chosen structure) and (strict) cartesian closed functors, i.e., functors $F: \mathcal{C} \rightarrow \mathcal{D}$ preserving binary products, projections, and the terminal object on the nose, and such that, for all objects $A, B \in \mathcal{C}$, currying

$$
F\left(B^{A}\right) \times F(A)=F\left(B^{A} \times A\right) \stackrel{F\left(e v_{A, B}\right)}{\longrightarrow} F(B)
$$

yields an identity. 
The functor $\mathcal{W}_{1}$ maps any cartesian closed category $\mathcal{C}$ to the signature with sorts $\mathcal{C}_{0}$, its set of objects, and with operations $A_{1}, \ldots, A_{n} \rightarrow A$ the set $\mathcal{C}\left(\llbracket A_{1} \times \ldots \times A_{n} \rrbracket, \llbracket A \rrbracket\right)$, where $\llbracket-\rrbracket$ denotes the function $\mathcal{L}_{0}\left(\mathcal{C}_{0}\right) \rightarrow \mathcal{C}_{0}$ defined by induction:

$$
\begin{array}{rlrl}
\llbracket c \rrbracket & =c & c \in \mathcal{C}_{0} \\
\llbracket 1 \rrbracket & =1 & \\
\llbracket A \times B \rrbracket & =\llbracket A \rrbracket \times \llbracket B \rrbracket & \\
\llbracket B^{A} \rrbracket & =\llbracket B \rrbracket^{\natural} \rrbracket .
\end{array}
$$

Conversely, given a 1-signature $X$, consider the simply-typed $\lambda$-calculus with base types in $X_{0}$ and constants in $X_{1}$. I.e., for any $c \in X_{1}(G, A)$ and terms $\Gamma \vdash M_{i}: A_{i}$, for all $1 \leq i \leq n$, where $G=\left(A_{1}, \ldots, A_{n}\right)$, we have a term $\Gamma \vdash c\left(M_{1}, \ldots, M_{n}\right): A$, representing the application of the constant $c$ to $M_{1}, \ldots, M_{n}$. We use special parentheses to avoid ambiguity with term application. Terms modulo $\beta \eta$ form a cartesian closed category $\mathcal{H}_{1}(X)$ with objects all types over $X_{0}$ and morphisms $A \rightarrow B$ all terms of type $B$ with one free variable of type $A$.

A less often formulated observation, which is useful to us, is that the adjunction $\mathcal{H}_{1} \dashv \mathcal{W}_{1}$ decomposes into two adjunctions

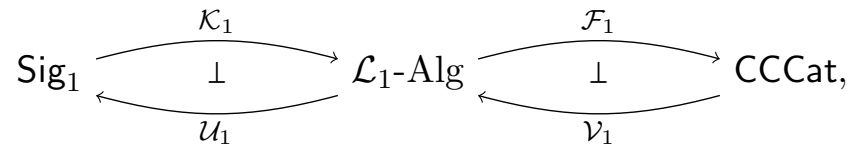

as follows.

Consider first the endofunctor $\mathcal{L}_{1}$ on $\operatorname{Sig}_{1}$ defined on objects by mapping any 1 -signature $X$ to the 1-signature with

- as sorts the set $X_{0}$, and

- as operations $\Gamma \vdash A$ the $\lambda$-terms $\Gamma \vdash M$ : $A$, with base types in $X_{0}$ and constants in $X_{1}$, as sketched above, modulo $\beta \eta$.

On morphisms of 1 -signatures $X \stackrel{f}{\rightarrow} Y$, let $\mathcal{L}_{1}(f)$ substitute constants $c \in X_{1}$ with $f_{1}(c)$. We obtain

Proposition 2.4. $\mathcal{L}_{1}$ is a monad on $\mathrm{Sig}_{1}$, with unit and multiplication, say $\eta$ and $\mu$.

Let now $\mathcal{L}_{1}$-Alg be the category of algebras for the monad $\mathcal{L}_{1}$ and $\mathcal{K}_{1}$ be the 'free algebra' functor $X \mapsto\left(\mathcal{L}_{1}(X), \mu_{X}\right)$.

The functor $\mathcal{V}_{1}$ maps any cartesian closed category $\mathcal{C}$ to the $\mathcal{L}_{1}$-algebra with base 1 signature $\left(\mathcal{C}_{0}, \mathcal{C}_{1}\right)$, defined as follows. First, $\mathcal{C}_{0}$ is the set of objects of $\mathcal{C}$. It has a canonical $\mathcal{L}_{0}$-algebra structure, say $h_{0}: \mathcal{L}_{0}\left(\mathcal{C}_{0}\right) \rightarrow \mathcal{C}_{0}$, obtained by interpreting type constructors in $\mathcal{C}$ as in (2.1). Extending this to contexts $G$ by $h_{0}(G)=\prod_{i} h_{0}\left(G_{i}\right)$, let the operations in $\mathcal{C}_{1}(G, A)$ be the morphisms in $\mathcal{C}\left(h_{0}(G), h_{0}(A)\right)$. Beware: the domain and codomain of such an operation are really $G$ and $A$, not $h_{0}(G)$ and $h_{0}(A)$. Similarly, interpreting the $\lambda$-calculus in $\mathcal{C}$, the 1 -signature $\left(\mathcal{C}_{0}, \mathcal{C}_{1}\right)$ has a canonical $\mathcal{L}_{1}$-algebra structure, say $h_{1}: \mathcal{L}_{1}\left(\mathcal{C}_{0}, \mathcal{C}_{1}\right) \rightarrow\left(\mathcal{C}_{0}, \mathcal{C}_{1}\right)$ :

$$
\begin{aligned}
h_{1}\left(G \vdash x_{i}: G_{i}\right) & =\pi_{i} \\
h_{1}(G \vdash(): 1) & =! \\
h_{1}\left(G \vdash c\left(M_{1}, \ldots, M_{n}\right): A\right) & =c \circ\left\langle h_{1}\left(M_{1}\right), \ldots, h_{1}\left(M_{n}\right)\right\rangle \\
h_{1}\left(G \vdash \lambda x: A \cdot M: B^{A}\right) & =\varphi\left(h_{1}(G, x: A \vdash M: B)\right) \\
h_{1}(G \vdash M N: B) & =e v \circ\left\langle h_{1}(M), h_{1}(N)\right\rangle \\
h_{1}(G \vdash(M, N): A \times B) & =\left\langle h_{1}(M), h_{1}(N)\right\rangle \\
h_{1}(G \vdash \pi M: A) & =\pi \circ h_{1}(M) \\
h_{1}\left(G \vdash \pi^{\prime} M: A\right) & =\pi^{\prime} \circ h_{1}(M),
\end{aligned}
$$


where ! is the unique morphism $h_{0}(G) \rightarrow 1, \varphi$ is the bijection $\mathcal{C}\left(h_{0}(G, A), h_{0}(B)\right) \cong$ $\mathcal{C}\left(h_{0}(G), h_{0}\left(B^{A}\right)\right)$, and $e v$ is the structure morphism $h_{0}\left(B^{A} \times A\right) \rightarrow h_{0}(B)$.

$\mathcal{L}_{1}$-algebras are much like cartesian closed categories whose objects are freely generated by their set of sorts. A perhaps useful analogy here is with multicategories $\mathcal{M}$, seen as being close to monoidal categories whose objects are freely generated by those of $\mathcal{M}$ by tensor and unit. Here, the functor $\mathcal{F}_{1}$ sends any $\mathcal{L}_{1}$-algebra $(X, h)$ to the cartesian closed category with

- objects the types over $X_{0}$, i.e., $\mathcal{L}_{0}\left(X_{0}\right)$,

- morphisms $A \rightarrow B$ the set of operations in $X_{1}(A, B)$.

This canonically forms a cartesian closed category, with structure induced by the $\mathcal{L}_{1}$-algebra structure. We define it in more detail in dimension 2 in Section 7.2 .

\section{Cartesian Closed 2-Signatures}

Given a 1-signature $X$, let $X_{\|}$denote the set of pairs of parallel operations, i.e., pairs of operations $M, N$ over the same sequent. Otherwise said, $X_{\|}$is the pullback

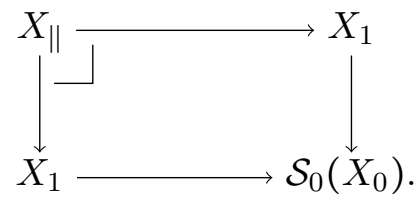

Any morphism $f: X \rightarrow Y$ of 1 -signatures yields a map $f_{\|}: X_{\|} \rightarrow Y_{\|}$, via the dashed arrow (obtained by universal property of pullback) in

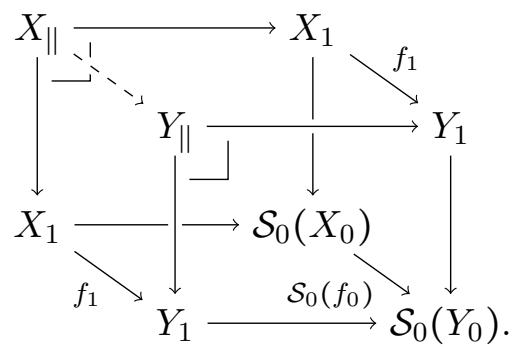

Definition 3.1. A 2-signature consists of a 1-signature $X$, plus a set $X_{2}$ of reduction rules with a map $X_{2} \rightarrow \mathcal{L}_{1}(X)_{\|}$.

A morphism of 2-signatures $\left(X, X_{2}\right) \rightarrow\left(Y, Y_{2}\right)$ is a pair $\left(f, f_{2}\right)$ where $f: X \rightarrow Y$ is a morphism of 1-signatures and $f_{2}: X_{2} \rightarrow Y_{2}$ makes the diagram

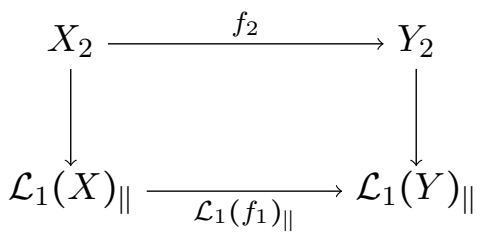

commute. We obtain:

Proposition 3.2. Composition of morphisms is associative and unital, and hence 2-signatures and their morphisms form a category Sig. 


\section{EXAMPLES}

4.1. Higher-order rewrite systems. The prime example of a 2-signature is that for the pure $\lambda$-calculus: it has a sort $t$ and operations

$$
a:(t, t \vdash t) \quad \ell:\left(t^{t} \vdash t\right),
$$

with a reduction rule $\beta$ over the pair $x: t^{t}, y: t \vdash a(\ell(x), y), x(y): t$ in $\mathcal{L}_{1}(\{t\},\{\ell, a\})_{\|}$. Categorically, this will yield a 2-cell

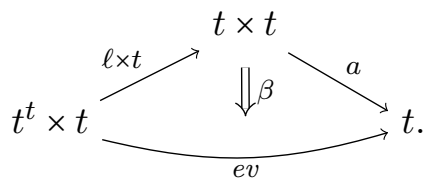

This is an example of a higher-order rewrite system in the sense of Nipkow [15]. Nipkow's definition is formally different, but his higher-order rewrite systems are in bijection with 2-signatures $h: X_{2} \rightarrow \mathcal{L}_{1}(X)_{\|}$such that for all rules $r \in X_{2}$, letting $(\Gamma \vdash M, N: A)=h(r)$ :

- $M$ is not a variable,

- $A$ is a sort,

- each variable occurring in $\Gamma$ occurs free in $M$.

These restrictions help dealing with decidability problems on higher-order rewrite systems, whose extension to our setting we leave open.

Let us now anticipate over Adjunction (1.1) and our main results below and state our soundness and completeness theorem. Given a higher-order rewrite system $X$, i.e., a 2 -signature satisfying the above conditions, let $\mathcal{R}(X)$ be the following locally-preordered 2-category. It has:

- objects are types in $\mathcal{L}_{0}\left(X_{0}\right)$;

- morphisms $A \rightarrow B$ are $\lambda$-terms in $\mathcal{L}_{1}(X)(A \vdash B)$, modulo $\beta \eta$;

- given two parallel morphisms $M$ and $N$, there is one 2-cell $M \rightarrow N$ exactly when there is a sequence of reductions $M \rightarrow^{*} N$ in the usual sense [15].

Proposition 4.1. $\mathcal{R}(X)$ is 2-cartesian closed.

$\mathcal{R}(X)$ and $\mathcal{H}(X)$ have the same objects and morphisms. But because our inference rules for forming reductions are the same as deduction rules for proving the existence of a reduction in the usual sense, we may map any reduction $P: M \rightarrow N$ to the unique reduction $M \rightarrow N$ in $\mathcal{R}(X)$. Conversely, any standard reduction step has a proof, which provides a reduction $P$. We have proved:

Theorem 4.2 (Soundness and completeness). There exists an identity-on-objects, identityon-morphisms, locally full cartesian closed 2-functor $\mathcal{H}(X) \stackrel{!}{\rightarrow} \mathcal{R}(X)$.

4.2. Theories with binding. Understanding reduction rules as equations, it is easy to define the free cartesian closed category generated by a 2 -signature. This yields the adjunction

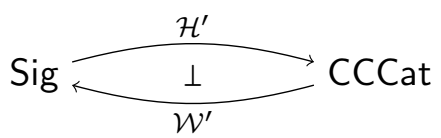

recalled above. 
This adjunction provides a categorical semantics for theories with binding, which is more general than other approaches by Fiore and Hur [7], Hirschowitz and Maggesi [9], and Zsidó [20].

If I understand correctly, the motivation for Fiore and Hur's subtle approach is the will to explain the $\lambda$-calculus by strictly less than itself. The present framework does not obey this specification, and instead tends to view the $\lambda$-calculus as a universal (parameterised) theory with binding.

We end this section by giving a formal construction of Adjunction (4.1). Cartesian closed categories form a full, reflective subcategory of 2CCCat, via the functor $\mathcal{J}: 2 \mathrm{CCC}$ at $\rightarrow$ CCCat mapping any cartesian closed 2-category $\mathcal{C}$ to the cartesian closed category with:

- objects those of $\mathcal{C}$,

- morphisms those of $\mathcal{C}$, modulo the congruence generated by $f \sim g$ iff there exists a 2-cell $f \rightarrow g$.

Here, $\mathcal{J}(\mathcal{C})$ is thought of as the free locally discrete cartesian closed 2-category. Adjunction (4.1) is obtained by composing the adjunctions

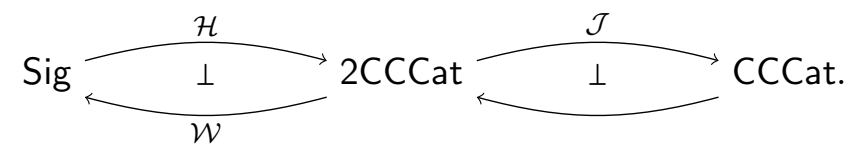

4.3. Non-examples. Non-examples are given by calculi whose reduction semantics is defined on terms modulo a so-called structural congruence, e.g., CCS [13], or the $\pi$-calculus [6, 14.

For example, consider the CCS term $(a \mid 0) \mid \bar{a}$. In CCS, it is structurally equivalent to $(a \mid \bar{a}) \mid 0$, which then reduces to $0 \mid 0$.

In order to account for this, we would have to consider a 2-signature with reduction rules for structural congruence, here $\left(M_{1} \mid M_{2}\right)\left|M_{3} \rightarrow M_{1}\right|\left(M_{2} \mid M_{3}\right)$ for associativity, and $M|N \rightarrow N| M$ for commutativity. But then, these reductions count as proper reductions, which departs from the desired computational behaviour. For example, the term $a \mid a$ has an infinite reduction sequence, using commutativity.

Anticipating the development in the next sections, a potential solution is to extend 2signatures to 2-theories. For any 2-signature $X$, let $X_{\|}$denote the set of pairs of reduction rules $r, s$ with a common type $G \vdash M \rightarrow N: A$. A 2-theory is a 2-signature $X$, together with a set of equations between parallel reductions, i.e., a subset $X_{3}$ of $\mathcal{L}(X)_{\|}$(where $\mathcal{L}$ is defined in Section 5 ).

Another possibility would be to define 2-theories to constist of a pair of sets over $\mathcal{L}_{1}(X)_{\|}$, one for structural equations, and the other for proper reduction rules.

The main adjunction announced above (1.1) extends to an adjunction between 2theories and cartesian closed 2-categories. Using equations, we may specify that any reduction $M \rightarrow M$ using only structural rules be the identity on $M$, and consider the computational behaviour of a 2-category to consist of its non-invertible 2-cells, as proposed by Hilken [8]. A question is whether for a given calculus this can be done with finitely many equations. 


\section{A 2-LAMBDA-CALCULUS}

We now begin the construction of Adjunction (1.1). We start in this section by defining a $\operatorname{monad} \mathcal{L}$ on Sig, which we will use to factor Adjunction (1.1) as

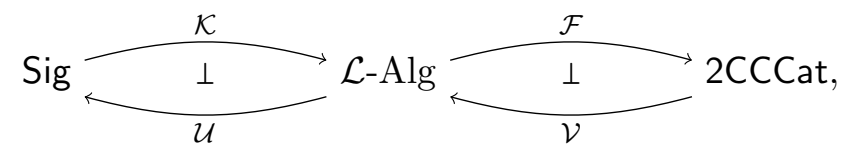

where:

- $\mathcal{L}$-Alg is the category of $\mathcal{L}$-algebras,

- $\mathcal{K}$ : Sig $\rightarrow \mathcal{L}$-Alg maps any $X$ to the free $\mathcal{L}$-algebra $\left(\mathcal{L}^{2} X \stackrel{\mu}{\rightarrow} \mathcal{L} X\right)$,

- $\mathcal{U}(\mathcal{L} X \stackrel{h}{\rightarrow} X)=X$,

- 2CCCat is the category of cartesian closed 2-categories, which we define in Section 6 .

The left-hand adjunction holds by $\mathcal{L}$ being a monad, thus we concentrate in Section 7 on establishing the right-hand one.

But for now, let us define the monad $\mathcal{L}$.

5.1. Syntax. Given a 2-signature $X=\left(\left(X_{0}, X_{1}\right), a: X_{2} \rightarrow \mathcal{L}_{1}(X)_{\|}\right)$(actually $\mathcal{L}_{1}(X)$ is $\left.\mathcal{L}_{1}\left(X_{0}, X_{1}\right)\right)$, we construct a new 2-signature $\mathcal{L}(X)$, whose reduction rules represent reduction sequences in the "higher-order rewrite system" defined by $X$, modulo permutation equivalence. The 2-signature $\mathcal{L}(X)$ has the same base 1 -signature $\left(X_{0}, X_{1}\right)$, and as reduction rules the terms of a $2 \lambda$-calculus (in the sense of Hilken [8]) modulo permutation equivalence, which we now define.

First, terms, called reductions, are defined by induction in Figure 1. The typing judgement has the shape $\Gamma \vdash P: M \rightarrow N: A$, where $A$ is a type in $\mathcal{L}_{0}\left(X_{0}\right), \Gamma$ is a list of pairs of a variable and a type, with no variable appearing more than once, $M$ and $N$ are terms of type $\Gamma \vdash A$ modulo $\beta \eta$, and $P$ is a reduction. In the sequel, we often forget the variables in such pairs $(\Gamma \vdash A)$, and identify them with sequents in $\mathcal{S}_{0}\left(X_{0}\right)$.

Remark 5.1. For any $\Gamma \vdash M: A$, we have a reduction $\Gamma \vdash M: M \rightarrow M: A$.

When clear from context, we abbreviate substitutions $\left[M_{1} / x_{1}, \ldots, M_{n} / x_{n}\right]$ of terms by $\left[M_{1}, \ldots, M_{n}\right]$. For a context $G, G_{i}$ denotes its $i$ th type. Also, for $(M, N) \in \mathcal{L}_{1}(X)_{\|}$, we let $X(M, N)$ be the set of all reduction rules $r \in X_{2}$ such that $a(r)=(M, N)$. We write $X(\Gamma \vdash M, N: A)$ to indicate the common type of $M$ and $N$. Similarly, $X(G \vdash A)$ denotes the set of operations in $X_{1}$ above $G \vdash A$.

5.2. Substitution. Next, we define substitution, which has "type"

$$
\frac{\Gamma \vdash Q: N \rightarrow N^{\prime}: \Delta \quad \Delta \vdash P: M \rightarrow M^{\prime}: A}{\Gamma \vdash P[Q]: M[N] \rightarrow M^{\prime}\left[N^{\prime}\right]: A,}
$$

i.e., given a reduction $P$ and a tuple of reductions $Q$, it produces a reduction of the indicated type, which we denote by $P[Q]$. Here, we denote by $\Gamma \vdash Q: N \rightarrow N^{\prime}: \Delta$ a tuple of reductions $\Gamma \vdash Q_{i}: N_{i} \rightarrow N_{i}^{\prime}: \Delta_{i}$, for $1 \leq i \leq|\Delta|$.

First, observe that we have a form of weakening: for any reduction $\Gamma \vdash P: M \rightarrow N: A$ and $x \notin \Gamma$, we also have $\Gamma, x: B \vdash P: M \rightarrow N: A$. We use this implicitly in the following.

The definition of substitution is a bit tricky: 


$$
\begin{aligned}
& \frac{\ldots \quad \Gamma \vdash P_{i}: M_{i} \rightarrow N_{i}: G_{i} \quad \ldots}{\Gamma \vdash r\left\langle\left\langle P_{1}, \ldots, P_{n}\right\rangle\right\rangle: M\left[M_{1}, \ldots, M_{n}\right] \rightarrow N\left[N_{1}, \ldots, N_{n}\right]: A}(r \in X(G \vdash M, N: A)) \\
& \frac{\Gamma \vdash P: M_{1} \rightarrow M_{2}: A \quad \Gamma \vdash Q: M_{2} \rightarrow M_{3}: A}{\Gamma \vdash P ; M_{2} Q: M_{1} \rightarrow M_{3}: A} \\
& \Gamma, x: A, \Delta \vdash x: x \rightarrow x: A \quad \Gamma \vdash():() \rightarrow(): 1 \\
& \frac{\Gamma \vdash P_{1}: M_{1} \rightarrow N_{1}: G_{1} \quad \ldots \quad \Gamma \vdash P_{n}: M_{n} \rightarrow N_{n}: G_{n}}{\Gamma \vdash c\left(P_{1}, \ldots, P_{n}\right): c\left(M_{1}, \ldots, M_{n}\right) \rightarrow c\left(N_{1}, \ldots, N_{n}\right): A}\left(c \in X_{1}(G \vdash A)\right) \\
& \frac{\Gamma, x: A \vdash P: M \rightarrow N: B}{\Gamma \vdash \lambda x: A . P: \lambda x: A . M \rightarrow \lambda x: A \cdot N: B^{A}} \quad \frac{\Gamma \vdash P: M \rightarrow M^{\prime}: B^{A} \quad \Gamma \vdash Q: N \rightarrow N^{\prime}: A}{\Gamma \vdash P Q: M N \rightarrow M^{\prime} N^{\prime}: B} \\
& \frac{\Gamma \vdash P: M \rightarrow M^{\prime}: A \quad \Gamma \vdash Q: N \rightarrow N^{\prime}: B}{\Gamma \vdash(P, Q):(M, N) \rightarrow\left(M^{\prime}, N^{\prime}\right): A \times B} \\
& \frac{\Gamma \vdash P: M \rightarrow N: A \times B}{\Gamma \vdash \pi_{A, B} P: \pi_{A, B} M \rightarrow \pi_{A, B} N: A} \quad \frac{\Gamma \vdash P: M \rightarrow N: A \times B}{\Gamma \vdash \pi_{A, B}^{\prime} P: \pi_{A, B}^{\prime} M \rightarrow \pi_{A, B}^{\prime} N: B}
\end{aligned}
$$

Figure 1: Reductions

- first we define left whiskering, which has "type"

$$
\frac{\Gamma \vdash Q: N \rightarrow N^{\prime}: \Delta \quad \Delta \vdash M: A}{\Gamma \vdash M[Q]: M[N] \rightarrow M^{\prime}\left[N^{\prime}\right]: A ;}
$$

- then we define right whiskering, which has "type"

$$
\frac{\Gamma \vdash N: \Delta \quad \Delta \vdash P: M \rightarrow M^{\prime}: A}{\Gamma \vdash P[N]: M[N] \rightarrow M^{\prime}[N]: A,}
$$

(where $N$ denotes a tuple);

- then we define substitution by $P[Q]=\left(P[N] ;_{M^{\prime}[N]} M^{\prime}[Q]\right)$.

There is of course another legitimate definition, namely $M[Q] ;_{M\left[N^{\prime}\right]} P\left[N^{\prime}\right]$. The two will be equated by permutation equivalence in the next section. by:

Left whiskering is defined inductively, with $\Delta=\left(x_{1}: A_{1}, \ldots, x_{n}: A_{n}\right)$ and $Q=\left(Q_{1}, \ldots, Q_{n}\right)$,

$$
\begin{aligned}
()[Q] & =() \\
x_{i}[Q] & =Q_{i} \\
c\left(M_{1}, \ldots, M_{p}\right)[Q] & =c\left(M_{1}[Q], \ldots, M_{p}[Q]\right) \\
(\lambda x: B . M)[Q] & =\lambda x: B .(M[Q, x]) \quad(\text { for } x \notin \operatorname{dom}(\Delta)) \\
(M N)[Q] & =(M[Q] N[Q]) \\
(M, N)[Q] & =(M[Q], N[Q]) \\
\left(\pi_{A, B} M\right)[Q] & =\pi_{A, B}(M[Q]) \\
\left(\pi_{A, B}^{\prime} M\right)[Q] & =\pi_{A, B}^{\prime}(M[Q])
\end{aligned}
$$


Right whiskering is defined inductively, with $\Delta=\left(x_{1}: A_{1}, \ldots, x_{n}: A_{n}\right)$ and $N=\left(N_{1}, \ldots, N_{n}\right)$, by:

$$
\begin{aligned}
\left(r\left\langle P_{1}, \ldots, P_{p}\right\rangle\right)[N] & \left.=r\left\langle P_{1}[N], \ldots, P_{p}[N]\right\rangle\right) \\
\left(P_{1} ; M^{\prime \prime} P_{2}\right)[N] & =\left(P_{1}[N] ; M^{\prime \prime}[N] P_{2}[N]\right) \\
()[N] & =() \\
x_{i}[N] & =N_{i} \\
c\left(P_{1}, \ldots, P_{p}\right)[N] & =c\left(P_{1}[N], \ldots, P_{p}[N]\right) \\
\left(\lambda x: B . P^{\prime}\right)[N] & =\lambda x: B .\left(P^{\prime}[N, x]\right) \quad(\text { for } x \notin \operatorname{dom}(\Delta)) \\
\left(P_{1} P_{2}\right)[N] & =\left(P_{1}[N] P_{2}[N]\right) \\
\left(P_{1}, P_{2}\right)[N] & =\left(P_{1}[N], P_{2}[N]\right) \\
\left(\pi_{A, B} P^{\prime}\right)[N] & =\pi_{A, B}\left(P^{\prime}[N]\right) \\
\left(\pi_{A, B}^{\prime} P^{\prime}\right)[N] & =\pi_{A, B}^{\prime}\left(P^{\prime}[N]\right) .
\end{aligned}
$$

Definition 5.2. Let $P[Q]=\left(P[N] ;_{M^{\prime}[N]} M^{\prime}[Q]\right)$.

Proposition 5.3. Given reductions $P$ and $Q$ as above, $P[Q]$ is a well-typed reduction $\Gamma \vdash P[Q]: M[N] \rightarrow M^{\prime}\left[N^{\prime}\right]: A$.

5.3. Permutation equivalence. We now define permutation equivalence on reductions, by the equations in Figures 3, 4 and 5, in Appendix A. The congruence rules in Figure 3 are bureaucratic: they just say that permutation equivalence is a congruence. The category rules make reductions of a given type $\Gamma \vdash A$ into a category. In Figure 4 , the beta and eta rules mirror the term-level beta and eta rules. Finally, the lifting rules lift composition of reductions towards toplevel.

So, $\mathcal{L}(X)$ has sorts $X_{0}$, operations $X_{1}$, and as reduction rules in $\mathcal{L}(X)(G \vdash M, N: A)$ all reductions $G \vdash P: M \rightarrow N: A$, modulo the equations.

This easily extends to:

Proposition 5.4. $\mathcal{L}$ is a functor $\mathrm{Sig} \rightarrow \mathrm{Sig}$.

Now, consider $\mathcal{L} \mathcal{L}(X)$. We define a mapping $\mu_{X}: \mathcal{L} \mathcal{L}(X) \rightarrow \mathcal{L}(X)$, by induction on reductions. The typing rule for reduction rules in $\mathcal{L} \mathcal{L}(X)$ specialises to:

$$
\frac{(R \in \mathcal{L}(X)(G \vdash M, N: A)) \quad \Gamma \vdash P_{1}: M_{1} \rightarrow N_{1}: G_{1} \quad \ldots \quad \quad \Gamma \vdash P_{n}: M_{n} \rightarrow N_{n}: G_{n}}{\Gamma \vdash R\left\langle\left\langle P_{1}, \ldots, P_{n}\right\rangle\right): M\left[M_{1}, \ldots, M_{n}\right] \rightarrow N\left[N_{1}, \ldots, N_{n}\right]: A} .
$$

We set $\mu\left(R\left\langle\left\langle P_{1}, \ldots, P_{n}\right\rangle\right\rangle\right)=R\left[\mu\left(P_{1}\right), \ldots, \mu\left(P_{n}\right)\right]$. The other cases just propagate the substitution:

$$
\begin{aligned}
P ; Q & \mapsto \mu(P) ; \mu(Q) \\
x & \mapsto x \\
() & \mapsto() \\
c\left(P_{1}, \ldots, P_{n}\right) & \mapsto c\left(\mu\left(P_{1}\right), \ldots, \mu\left(P_{n}\right)\right) \\
\lambda x: A . P & \mapsto \lambda x: \mu(P) \\
P Q & \mapsto \mu(P) \mu(Q) \\
(P, Q) & \mapsto(\mu(P), \mu(Q)) \\
\pi P & \mapsto \pi(\mu(P)) \\
\pi^{\prime} P & \mapsto \pi^{\prime}(\mu(P)) .
\end{aligned}
$$

Lemma 5.5. This defines a natural transformation $\mu: \mathcal{L}^{2} \rightarrow \mathcal{L}$, which makes the diagram 


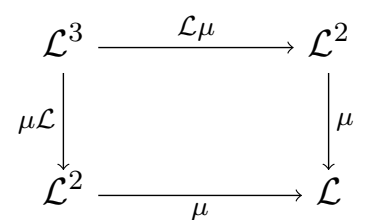

commute.

Similarly, there is a natural transformation $\eta: i d \rightarrow \mathcal{L}$, mapping each $r \in X(G \vdash M, N: A)$ to the reduction $G \vdash r\left\langle\left\langle x_{1}, \ldots, x_{n}\right\rangle\right\rangle: M \rightarrow N: A$, and we have:

Lemma 5.6. The diagram

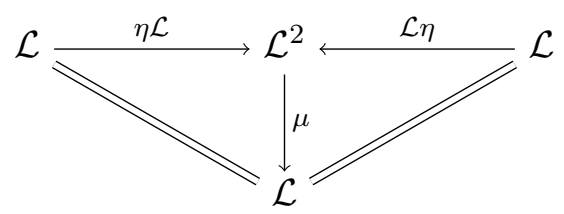

commutes.

Corollary 5.7. $(\mathcal{L}, \mu, \eta)$ is a monad on Sig.

A crucial result is:

Proposition 5.8. For all $\Gamma \vdash Q: N \rightarrow N^{\prime}: \Delta$ and $\Delta \vdash P: M \rightarrow M^{\prime}: A$, we have:

$$
\Gamma \vdash P[Q] \equiv\left(M[Q] ;_{M\left[N^{\prime}\right]} P\left[N^{\prime}\right]\right): M[N] \rightarrow M^{\prime}\left[N^{\prime}\right]: A .
$$

Proof. We proceed by induction on $P$. Most cases are bureaucratic. Consider for instance $P=c\left(P_{1}, \ldots, P_{p}\right)$. Then, by definition:

$$
P[Q]=\left(c\left(P_{1}[N], \ldots, P_{p}[N]\right) ; c\left(M_{1}^{\prime}[N], \ldots, M_{p}^{\prime}[N]\right) c\left(M_{1}^{\prime}[Q], \ldots, M_{p}^{\prime}[Q]\right) .\right.
$$

By the third lifting rule, this is permutation equivalent to

$$
c\left(P_{1}[N] ;_{M_{1}^{\prime}[N]} M_{1}^{\prime}[Q], \ldots, P_{p}[N] ;_{M_{p}^{\prime}[N]} M_{p}^{\prime}[Q]\right) .
$$

By $p$ applications of the induction hypothesis, we obtain

$$
c\left(M_{1}[Q] ;_{M_{1}\left[N^{\prime}\right]} P_{1}\left[N^{\prime}\right], \ldots, M_{p}[Q] ;_{M_{p}\left[N^{\prime}\right]} P_{p}\left[N^{\prime}\right]\right),
$$

which by lifting again yields the desired result:

$$
c\left(M_{1}[Q], \ldots, M_{p}[Q]\right) ; c\left(M_{1}\left[N^{\prime}\right], \ldots, M_{p}\left[N^{\prime}\right]\right) c\left(P_{1}\left[N^{\prime}\right], \ldots, P_{p}\left[N^{\prime}\right]\right) .
$$

The case where something actually happens is $P=r\left\langle\left\langle P_{1}, \ldots, P_{p}\right\rangle\right\rangle$, with $r \in X(G \vdash$ $\left.M_{0}, M_{0}^{\prime}: A\right)$ and each $\Delta \vdash P_{i}: M_{i} \rightarrow M_{i}^{\prime}: G_{i}$. Then, the left-hand side is

$$
r\left\langle\left\langle P_{1}[N], \ldots, P_{p}[N]\right\rangle ; M_{0}^{\prime}\left[M_{1}^{\prime}, \ldots, M_{n}^{\prime}\right][N] M_{0}^{\prime}\left[M_{1}^{\prime}, \ldots, M_{p}^{\prime}\right][Q] .\right.
$$

By lifting, omitting indices of vertical compositions and implicitly using the category rules, we have

$$
r\left\langle\langle P _ { 1 } [ N ] , \ldots , P _ { p } [ N ] \rangle \equiv r \left\langle\left\langle M_{1}[N], \ldots, M_{p}[N]\right\rangle ; M_{0}^{\prime}\left[P_{1}[N], \ldots, P_{p}[N]\right] .\right.\right.
$$

Observing that $M_{0}^{\prime}\left[M_{1}^{\prime}, \ldots, M_{p}^{\prime}\right][Q]=M_{0}^{\prime}\left[M_{1}^{\prime}[Q], \ldots, M_{p}^{\prime}[Q]\right]$, the whole is permutation equivalent to

$$
\begin{aligned}
& r\left\langle\left\langle M_{1}[N], \ldots, M_{p}[N]\right\rangle ;\right. \\
& M_{0}^{\prime}\left[P_{1}[N], \ldots, P_{p}[N]\right] \\
& M_{0}^{\prime}\left[M_{1}^{\prime}[Q], \ldots, M_{p}^{\prime}[Q]\right]
\end{aligned}
$$


i.e., by lifting (inductively):

$$
\begin{aligned}
& r\left\langle M_{1}[N], \ldots, M_{p}[N]\right\rangle ; \\
& M_{0}^{\prime}\left[\left(P_{1}[N] ; M_{1}^{\prime}[Q]\right), \ldots,\left(P_{p}[N] ; M_{p}^{\prime}[Q]\right)\right] .
\end{aligned}
$$

By induction hypothesis, this is permutation equivalent to

$$
\begin{aligned}
& r\left\langle M_{1}[N], \ldots, M_{p}[N]\right\rangle ; \\
& M_{0}^{\prime}\left[\left(M_{1}[Q] ; P_{1}\left[N^{\prime}\right]\right), \ldots,\left(M_{p}[Q] ; P_{p}\left[N^{\prime}\right]\right)\right],
\end{aligned}
$$

i.e., by lifting again to

$$
\begin{aligned}
& r\left\langle\left\langle M_{1}[N], \ldots, M_{p}[N]\right\rangle ;\right. \\
& M_{0}^{\prime}\left[M_{1}[Q], \ldots, M_{p}[Q]\right] ; \\
& M_{0}^{\prime}\left[P_{1}\left[N^{\prime}\right], \ldots, P_{p}\left[N^{\prime}\right]\right] .
\end{aligned}
$$

The second lifting rule then yields

$$
\begin{aligned}
& r\left\langle\left\langle M_{1}[Q], \ldots, M_{p}[Q]\right\rangle ;\right. \\
& M_{0}^{\prime}\left[P_{1}\left[N^{\prime}\right], \ldots, P_{p}\left[N^{\prime}\right]\right]
\end{aligned}
$$

and hence

$$
\begin{aligned}
& M_{0}\left[M_{1}[Q], \ldots, M_{p}[Q]\right] ; \\
& r\left\langle M_{1}\left[N^{\prime}\right], \ldots, M_{p}\left[N^{\prime}\right]\right\rangle ; \\
& M_{0}^{\prime}\left[P_{1}\left[N^{\prime}\right], \ldots, P_{p}\left[N^{\prime}\right]\right],
\end{aligned}
$$

so, by the second lifting rule again:

$$
\begin{aligned}
& M_{0}\left[M_{1}[Q], \ldots, M_{p}[Q]\right] \\
& r\left\langle\left\langle P_{1}\left[N^{\prime}\right], \ldots, P_{p}\left[N^{\prime}\right]\right\rangle,\right.
\end{aligned}
$$

i.e., the right-hand side.

\section{Cartesian Closed 2-CATEgories}

6.1. Definition. In a 2-category $\mathcal{C}$, a diagram $A \stackrel{p}{\leftarrow} C \stackrel{q}{\rightarrow} B$ is a product diagram iff for all object $D$, the induced functor

$$
\mathcal{C}(D, C) \stackrel{\langle\mathcal{C}(D, p), \mathcal{C}(D, q)\rangle}{\longrightarrow} \mathcal{C}(D, A) \times \mathcal{C}(D, B)
$$

is an isomorphism of categories. Because this family of functors is 2-natural in $D$, the inverse functors will also be 2-natural.

Similarly, an object 1 of $\mathcal{C}$ is terminal iff for all $D$ the unique functor

$$
\mathcal{C}(D, 1) \stackrel{!}{\rightarrow} 1
$$

is an isomorphism (where the right-hand 1 is the terminal category).

Definition 6.1. A 2-category with finite products, or fp 2-category, is a 2-category $\mathcal{C}$, equipped with a terminal object and a 2 -functor

$$
\mathcal{C} \times \mathcal{C} \stackrel{\times}{\rightarrow} \mathcal{C}
$$

plus, for all $A$ and $B$, a product diagram

$$
A \stackrel{p}{\leftarrow} A \times B \stackrel{q}{\rightarrow} B .
$$

In such an fp 2-category $\mathcal{C}$, given objects $A$ and $B$, an exponential for them is a pair of an object $B^{A}$ and a morphism $e v: A \times B^{A} \rightarrow B$, such that for all $D$, the functor 


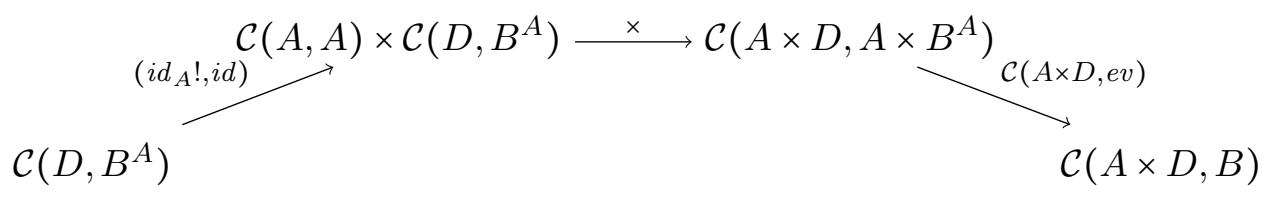

is an isomorphism. As above, because this family of functors is 2-natural in $D$, the inverse functors will also be 2-natural.

Definition 6.2. A cartesian closed 2-category, or cartesian closed 2-category, is an fp 2-category, equipped with a choice of exponentials for all pairs of objects. The category 2CCCat has cartesian closed 2-categories as objects, and stricly structure-preserving functors between them as morphisms.

We observe in particular that this implies preservation of projections and evaluation morphisms.

\section{MAin ADJUnCtion}

7.1. Right adjoint. Given a cartesian closed 2-category $\mathcal{C}$, define $\mathcal{V}(\mathcal{C})=\left(\mathcal{C}_{0}, \mathcal{C}_{1}\right.$, $\left.\mathcal{C}_{2}\right)$ as follows. First, let as in Section $2\left(\mathcal{C}_{0}, \mathcal{C}_{1}\right)=\mathcal{V}_{1}(\mathcal{C})$, and recall the canonical $\mathcal{L}_{0}$ and $\mathcal{L}_{1}$-algebra structures $h_{0}$ and $h_{1}$. Let then the reduction rules in $\mathcal{C}_{2}(G \vdash M, N: A)$ be the 2-cells in $\mathcal{C}\left(h_{0}(G), h_{0}(A)\right)\left(h_{1}(M), h_{1}(N)\right)$, abbreviated to $\mathcal{C}(G, A)(M, N)$ in the sequel.

This signature $\mathcal{V C}$ has a canonical $\mathcal{L}$-algebra structure $h_{2}: \mathcal{L}(\mathcal{V C}) \rightarrow \mathcal{V C}$, which we define by induction over reductions in Figure 2, In the case for $\lambda, \varphi$ denotes the structure isomorphism $\mathcal{C}((\Pi \Gamma) \times A, B) \cong \mathcal{C}\left(\Pi \Gamma, B^{A}\right)$.

In order for the definition to make sense as a morphism $\mathcal{L}(\mathcal{V C}) \rightarrow \mathcal{V C}$, we have to check its compatibility with the equations. We have first:

Lemma 7.1. For all $\Delta \vdash Q: N \rightarrow N^{\prime}: \Gamma$ and $\Gamma \vdash P: M \rightarrow M^{\prime}: A$ in $\mathcal{L}(\mathcal{V C})$,

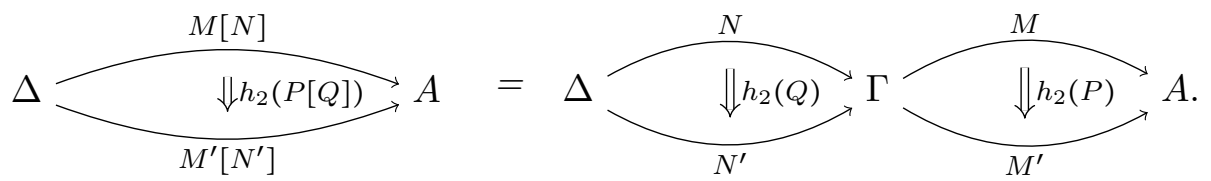

Proof. By induction on $P$ and the axioms for cartesian closed 2-categories.

Lemma 7.2. Any two equated reductions are mapped to the same 2-cell in $\mathcal{C}$.

Proof. We proceed by induction on the proof of the considered equation. The congruence and category rules of Figures 3 and 4 hold because, in $\mathcal{C}$, vertical composition is associative and unital, and equality is a congruence. The beta rule is less easy, so we spell it out.

The left-hand reduction is interpreted in $\mathcal{C}$ as

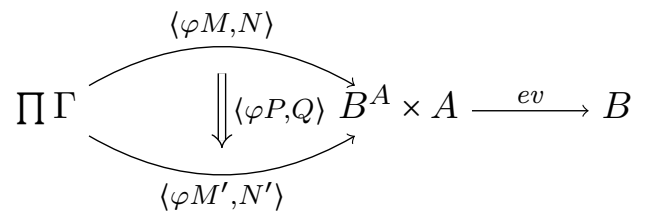

which is equal to 


$$
\begin{aligned}
& \left(G \vdash x_{i}: x_{i} \rightarrow x_{i}: G_{i}\right) \mapsto\left(i d_{\pi_{i}}: \pi_{i} \rightarrow \pi_{i}: \prod G \rightarrow G_{i}\right) \\
& (G \vdash():() \rightarrow(): 1) \mapsto\left(i d_{!}: ! \rightarrow !: \prod G \rightarrow 1\right) \\
& \left(\Gamma \vdash c\left(P_{1}, \ldots, P_{n}\right): c\left(M_{1}, \ldots, M_{n}\right) \rightarrow c\left(N_{1}, \ldots, N_{n}\right): A\right) \mapsto \\
& \left\langle M_{1}, \ldots, M_{n}\right\rangle \\
& \Pi \Gamma \underset{\left\langle N_{1}, \ldots, N_{n}\right\rangle}{\|_{P}} \Pi G \stackrel{c}{\longrightarrow} A \quad\left(c \in \mathcal{C}_{1}(G, A), P=\left\langle P_{1}, \ldots, P_{n}\right\rangle\right) \\
& \left(\Gamma \vdash r\left\langle\left\langle P_{1}, \ldots, P_{n}\right\rangle\right\rangle: M\left[M_{1}, \ldots, M_{n}\right] \rightarrow N\left[N_{1}, \ldots, N_{n}\right]: A\right) \mapsto
\end{aligned}
$$

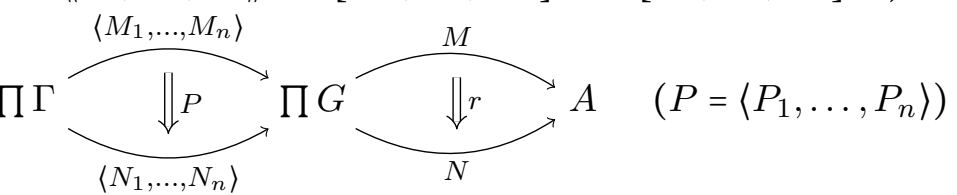

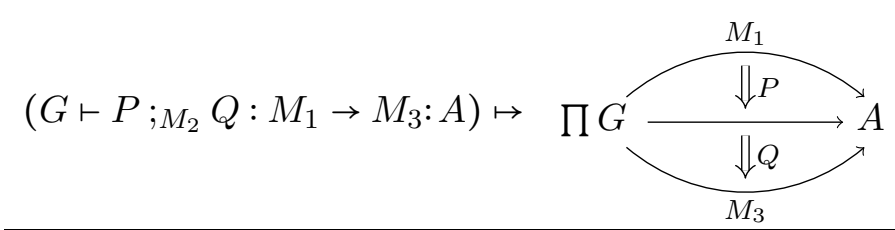

$$
\begin{aligned}
& \left(\Gamma \vdash \lambda x: A . P: \lambda x: A . M \rightarrow \lambda x: A . N: B^{A}\right) \mapsto \varphi(P: M \rightarrow N:(\Pi \Gamma) \times A \rightarrow B) \\
& \left(\Gamma \vdash P Q: M N \rightarrow M^{\prime} N^{\prime}: B\right) \mapsto \Pi \Gamma \overbrace{\downarrow\left\langle M^{\prime}, N^{\prime}\right\rangle}^{\langle M, N\rangle} \stackrel{\left\langle B^{A}\right.}{\longrightarrow} \times A \stackrel{e v}{\longrightarrow} B \\
& \left(\Gamma \vdash(P, Q):(M, N) \rightarrow\left(M^{\prime}, N^{\prime}\right): A \times B\right) \mapsto \prod_{\substack{\left\langle M^{\prime}, N^{\prime}\right\rangle}}^{\langle M, N\rangle} \\
& \left(\Gamma \vdash \pi_{A, B} P: \pi_{A, B} M \rightarrow \pi_{A, B} N: A\right) \mapsto \prod \Gamma \overbrace{\Downarrow_{P}}^{M} A \times B \stackrel{\pi}{\longrightarrow} A \\
& \left(\Gamma \vdash \pi_{A, B}^{\prime} P: \pi_{A, B}^{\prime} M \rightarrow \pi_{A, B}^{\prime} N: B\right) \mapsto \prod \Gamma \overbrace{N P}^{M} A \times B \stackrel{\pi^{\prime}}{\longrightarrow} B
\end{aligned}
$$

Figure 2: The $\mathcal{L}$-algebra structure on $\mathcal{V}(\mathcal{C})$ 


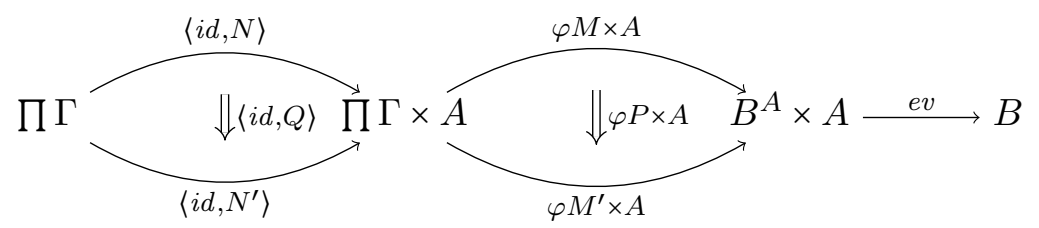

which is in turn equal (by cartesian closedness of $\mathcal{C}$ ) to:

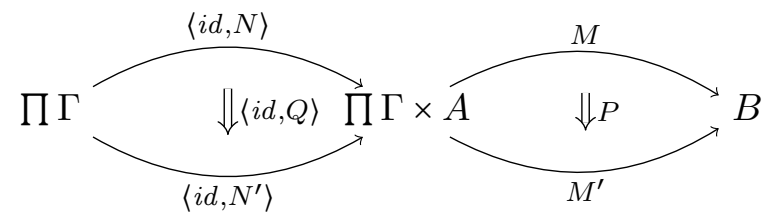

and hence to the right-hand side of the equation by Lemma 7.1. The other beta and eta rules similarly hold by the properties of products, internal homs, and terminal object in $\mathcal{C}$.

The lifting rules hold by (particular cases of) the interchange law in $\mathcal{C}$ and functoriality of the structural isomorphisms

$$
\mathcal{C}(A \times B, C) \cong \mathcal{C}\left(B, C^{A}\right) \quad \text { and } \quad \mathcal{C}(C, A \times B) \cong \mathcal{C}(C, A) \times \mathcal{C}(C, B),
$$

which concludes the proof.

This assignment extends to cartesian closed functors and we have:

Proposition 7.3. $\mathcal{V}$ is a functor 2CCCat $\rightarrow$ Sig.

7.2. Left adjoint. Given an $\mathcal{L}$-algebra $h: \mathcal{L}(X) \rightarrow X$, we now construct a cartesian closed 2-category $\mathcal{F}(X, h)$. It has:

- objects the types in $\mathcal{L}_{0}\left(X_{0}\right)$;

- 1-cells $A \rightarrow B$ the terms in $\mathcal{L}_{1}\left(X_{0}, X_{1}\right)(A, B)$;

- 2-cells $M \rightarrow N: A \rightarrow B$ the reduction rules in $X_{2}(M, N)$.

We then must define the cartesian closed 2-category structure, and we start with the 2category structure. Composition of 1-cells $A \stackrel{M}{\longrightarrow} B \stackrel{N}{\longrightarrow} C$ is defined to be $A \stackrel{N[M]}{\longrightarrow} C$. Identities are given by variables, as usual. Vertical composition of 2-cells

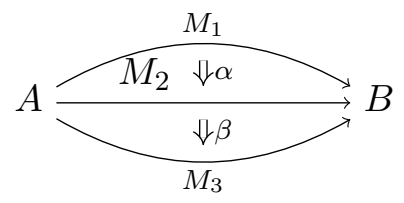

is given by $h\left(\eta(\alpha) ;_{M_{2}} \eta(\beta)\right)$.

Horizontal composition of 2-cells

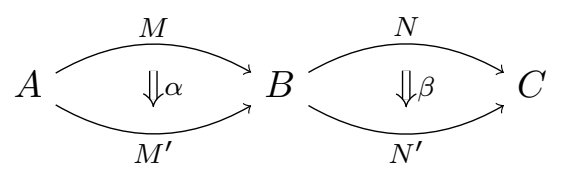

is obtained as $h(\beta\langle\eta \eta(\alpha)\rangle)$.

The identity at $A \stackrel{M}{\longrightarrow} B$ is $h(M)$. 
To show that this yields a 2-category structure, the only non obvious point is the interchange law. We deal with it using the following series of results. First, consider the left whiskering

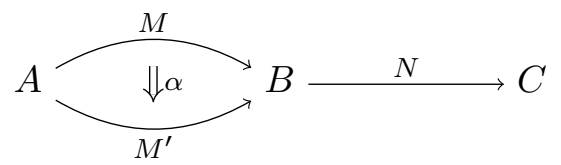

of a 2-cell $\alpha$ by a 1-cell $N$, i.e., the composition $i d_{N} \circ \alpha=h((h(N))\langle\langle\eta(\alpha)\rangle)$.

Lemma 7.4. We have: $h((h(N))\langle\langle\eta(\alpha)\rangle)=h(N[\eta(\alpha)])$.

Proof. Indeed, consider the term $N\langle\eta \eta(\eta(\alpha))\rangle$ in $\mathcal{L}(\mathcal{L}(X))$. Its images by $h \circ \mathcal{L}(h)$ and $h \circ \mu$ coincide, and are respectively $h\left((h(N))\langle\langle\eta(\alpha)\rangle)\right.$, i.e., $i d_{N} \circ \alpha$, and $h(N[\eta(\alpha)])$.

Similarly, consider the right whiskering

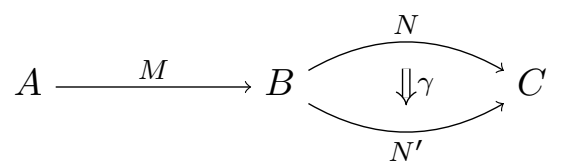

of a 2-cell $\gamma$ by a 1-cell $M$, i.e., the composition $\gamma \circ i d_{N}=h(\gamma\langle\langle\eta(h(M))\rangle\rangle)$.

Lemma 7.5. We have: $h(\gamma\langle\langle\eta(h(M))\rangle)=h(\gamma\langle\langle M\rangle)$.

Proof. Consider $(\eta \gamma)\langle\eta \eta M\rangle\rangle$ in $\mathcal{L}(\mathcal{L}(X))$. Its images by $h \circ \mathcal{L}(h)$ and $h \circ \mu$ coincide, and are respectively $h(\gamma\langle\langle\eta(h(M))\rangle\rangle)$ and $h(\gamma\langle\langle M\rangle)$.

Now, we prove that the two sensible ways of mimicking horizontal composition using whiskering coincide with actual horizontal composition:

Lemma 7.6. For any cells as in (7.1),

$$
\left(\beta \circ i d_{M}\right) ;\left(i d_{N^{\prime}} \circ \alpha\right)=\beta \circ \alpha=\left(i d_{N} \circ \alpha\right) ;\left(\beta \circ i d_{M^{\prime}}\right) \text {. }
$$

Proof. Consider first the reduction $\eta\left(\beta\langle\langle M\rangle) ; \eta\left(N^{\prime}[\eta(\alpha)]\right)\right.$ in $\mathcal{L}(\mathcal{L}(X))$. Taking $h \circ \mathcal{L}(h)$ and $h \circ \mu$ as above respectively yields

- $h\left(\eta\left(h(\beta\langle\langle M\rangle)) ; \eta\left(h\left(N^{\prime}[\eta(\alpha)]\right)\right)\right)\right.$, and

- $h\left(\beta\left\langle\langle M\rangle ; N^{\prime}[\eta(\alpha)]\right)=h(\beta\langle\eta(\alpha)\rangle)\right.$,

hence the left-hand equality. Then consider $\eta(N[\eta(\alpha)]) ; \eta\left(\beta\left(M^{\prime}\right)\right)$. Evaluating as before yields the right-hand equality.

Furthermore, consider any configuration like:

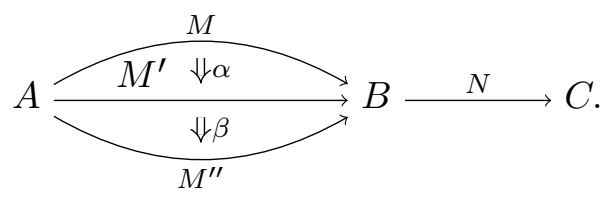

Lemma 7.7. We have $\left(i d_{N} \circ \alpha\right) ;\left(i d_{N} \circ \beta\right)=i d_{N} \circ(\alpha ; \beta)$.

Proof. Consider $\eta(N[\eta(\alpha)]) ; \eta(N[\eta(\beta)])$. Evaluating yields equality of

- $h(\eta(h(N[\eta(\alpha)])) ; \eta(h(N[\eta(\beta)])))$, i.e., the left-hand side, and

- $h(N[\eta(\alpha)] ; N[\eta(\beta)])$, i.e., $h(N[\eta(\alpha) ; \eta(\beta)])$ by lifting. 
But now consider $N[\eta(\eta(\alpha) ; \eta(\beta))]$. Evaluating yields equality of

- $h(N[\eta(\alpha) ; \eta(\beta)])$, as above, and

- $h(N[\eta(h(\eta(\alpha) ; \eta(\beta)))])$, i.e., $h(N[\eta(\alpha ; \beta)])$ (where $\alpha ; \beta$ denotes vertical composition in our candidate 2 -category), i.e., the right-hand side.

Finally, by a similar argument, we have:

Lemma 7.8. For any

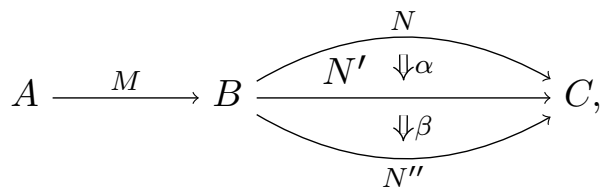

we have $\left(\alpha \circ i d_{M}\right) ;\left(\beta \circ i d_{M}\right)=(\alpha ; \beta) \circ i d_{M}$.

Lemma 7.9. The interchange law holds, i.e., for all reduction rules as in

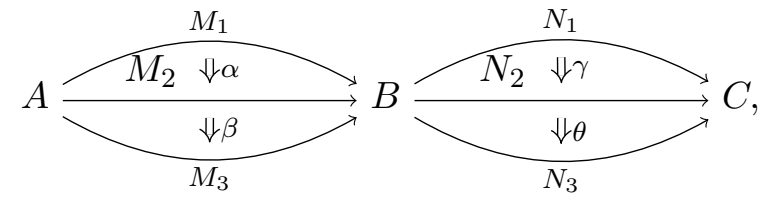

we have

$$
(\gamma ; \theta) \circ(\alpha ; \beta)=(\gamma \circ \alpha) ;(\theta \circ \beta) .
$$

Proof. By the previous results, we have

$$
\begin{aligned}
& (\gamma ; \theta) \circ(\alpha ; \beta) \\
& =\left((\gamma ; \theta) \circ M_{1}\right) ;\left(N_{3} \circ(\alpha ; \beta)\right) \\
& =\left(\gamma \circ M_{1}\right) ;\left(\theta \circ M_{1}\right) ;\left(N_{3} \circ \alpha\right) ;\left(N_{3} \circ \beta\right) \\
& =\left(\gamma \circ M_{1}\right) ;\left(N_{2} \circ \alpha\right) ;\left(\theta \circ M_{2}\right) ;\left(N_{3} \circ \beta\right) \\
& =(\gamma \circ \alpha) ;(\theta \circ \beta) .
\end{aligned}
$$

Now, let us show cartesian closedness. We have a bijection of hom-sets $\mathcal{L}_{1}(X)(C \vdash$ $A \times B) \cong \mathcal{L}_{1}(X)(C \vdash A) \times \mathcal{L}_{1}(X)(C \vdash B)$, given by

$$
\begin{aligned}
\mathcal{L}_{1}(X)(C \vdash A \times B) & \rightarrow \mathcal{L}_{1}(X)(C \vdash A) \times \mathcal{L}_{1}(X)(C \vdash B) \\
M & \mapsto \pi M, \pi^{\prime} M
\end{aligned}
$$

and

$$
\begin{aligned}
\mathcal{L}_{1}(X)(C \vdash A) \times \mathcal{L}_{1}(X)(C \vdash B) & \rightarrow \mathcal{L}_{1}(X)(C \vdash A \times B) \\
M, N & \mapsto(M, N) .
\end{aligned}
$$

These are mutually inverse thanks to the beta and eta rules for products in the simply-typed $\lambda$-calculus.

On 2-hom-sets, we have

$$
\begin{aligned}
\mathcal{L}(X)(C \vdash M, N: A \times B) & \rightarrow \mathcal{L}(X)(C \vdash \pi M, \pi N: A) \times \mathcal{L}(X)\left(C \vdash \pi^{\prime} M, \pi^{\prime} N: B\right) \\
P & \mapsto \pi P, \pi^{\prime} P
\end{aligned}
$$

and (omitting $C$ )

$$
\begin{aligned}
\mathcal{L}(X)\left(M_{1}, N_{1}: A\right) \times \mathcal{L}(X)\left(M_{2}, N_{2}: B\right) & \rightarrow \mathcal{L}(X)\left(\left(M_{1}, M_{2}\right),\left(N_{1}, N_{2}\right): A \times B\right) \\
P_{1}, P_{2} & \mapsto\left(P_{1}, P_{2}\right),
\end{aligned}
$$


which are mutually inverse thanks to the beta and eta rules for products in Figure 4. We use these to define the desired isomorphism $(u, v)$

$$
X_{2}(C \vdash M, N: A \times B) \cong X_{2}(C \vdash \pi M, \pi N: A) \times X_{2}\left(C \vdash \pi^{\prime} M, \pi^{\prime} N: B\right),
$$

as in the diagrams

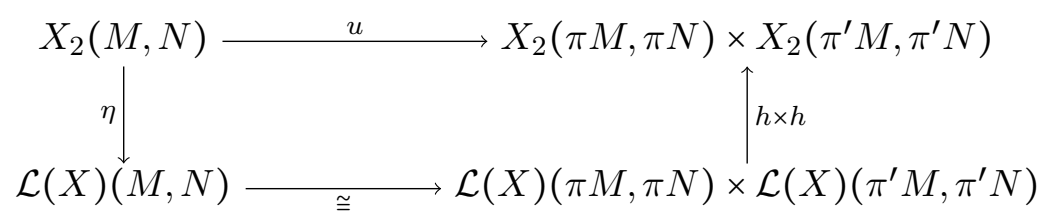

and

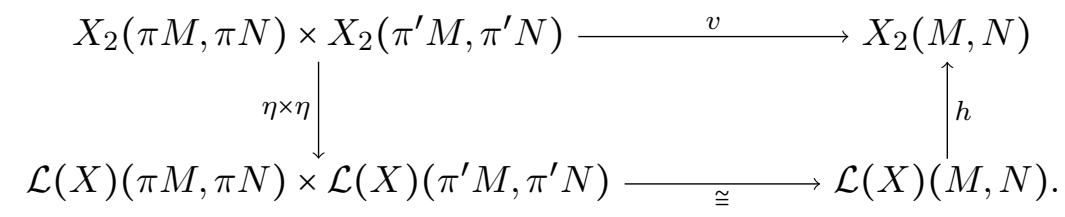

Starting from $r \in X_{2}(M, N)$, we obtain

$$
v(u(r))=h\left(\eta(h(\pi(\eta(r)))), \eta\left(h\left(\pi^{\prime}(\eta(r))\right)\right)\right) .
$$

But consider $\left(\eta(\pi \eta(r)), \eta\left(\pi^{\prime} \eta(r)\right)\right)$ in $\mathcal{L}(\mathcal{L} X)$; its images by $h \circ \mathcal{L} h$ and $h \circ \mu$ are respectively:

- $h\left(\eta(h(\pi(\eta r))), \eta\left(h\left(\pi^{\prime}(\eta r)\right)\right)\right)$, and

- $h\left(\pi \eta(r), \pi^{\prime} \eta(r)\right)$, i.e., $h(\eta(r))$, i.e., $r$,

which must be equal because $h$ is an $\mathcal{L}$-algebra, hence $v \circ u=i d$.

Conversely, starting from $(r, s) \in X_{2}\left(M_{1}, M_{2}\right) \times X_{2}\left(N_{1}, N_{2}\right)$, we obtain the pair with components

$$
h(\pi(\eta(h(\eta(r), \eta(s))))) \quad \text { and } \quad h\left(\pi^{\prime}(\eta(h(\eta(r), \eta(s))))\right) .
$$

Considering $\pi(\eta(\eta(r), \eta(s))) \in \mathcal{L}(\mathcal{L}(X))$, its images by $h \circ \mathcal{L}(h)$ and $h \circ \mu$ are respectively:

- $h(\pi(\eta(h(\eta(r), \eta(s)))))$, and

- $h(\pi(\eta(r), \eta(s)))=h(\eta(r))=r$.

As above, they must be equal, and by symmetry the second component is $s$, and we have proved $u \circ v=i d$. Similar reasoning for the terminal object and internal homs leads to:

Proposition 7.10. This yields a cartesian closed 2-category structure on $\mathcal{C}$.

This extends to morphisms of $\mathcal{L}$-algebras, so we have constructed a functor $\mathcal{F}: \mathcal{L}$-Alg $\rightarrow$ 2CCCat.

7.3. Adjunction. Consider any $\mathcal{L}$-algebra $(X, h)$. What does $(Y, k)=\mathcal{V}(\mathcal{F}(X, h))$ look like? Sorts in $Y_{0}$ are types in $\mathcal{L}_{0}\left(X_{0}\right)$. Operations $Y_{1}(G \vdash A)$ are terms in $\mathcal{L}_{1}\left(X_{0}, X_{1}\right)(\mu(\Pi G) \vdash$ $\mu(A)$ ), where $\mu$ denotes the monad multiplication for $\mathcal{L}_{0}$. Reduction rules in $Y_{2}(G \vdash$ $M, N: B)$ are reduction rules in $X_{2}\left(\mu(\Pi G) \vdash M^{\prime}, N^{\prime}: \mu(B)\right)$, where $M^{\prime}=M\left[\pi_{1} x / x_{1}, \ldots, \pi_{n} x / x_{n}\right]$ (and similarly for $N^{\prime}$ ).

Let $\eta_{X}^{\mathcal{L}}$ map:

- each sort $\iota \in X_{0}$ to the type $\iota \in \mathcal{L}_{0}\left(X_{0}\right)$ (this is the monad unit for $\mathcal{L}_{0}$ ),

- each operation $c \in X(G \vdash A)$ to the term $c\left(\pi_{1} x, \ldots, \pi_{n} x\right)$, and 
- each reduction rule $r \in X_{2}(G \vdash M, N: A)$ to the reduction rule

$$
h\left(r\left\langle\pi_{1} x, \ldots, \pi_{n} x\right\rangle\right) \in X_{2}\left(\prod G \vdash M^{\prime}, N^{\prime}: A\right) .
$$

(Thanks to the fact that $\mu\left(\mathcal{L}_{0}(\eta)(A)\right)=A$.)

Theorem 7.11. This $\eta^{\mathcal{L}}$ is a natural transformation which is the unit of an adjunction

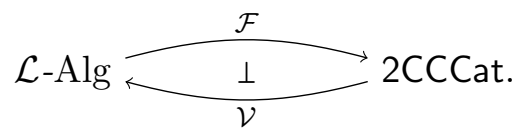

Proof. Consider any morphism $f:(X, h) \rightarrow \mathcal{V}(\mathcal{C})$, and let $\mathcal{X}=\mathcal{F}(X, h),(Y, k)=\mathcal{V}(\mathcal{X})$, and $\mathcal{V}(\mathcal{C})=\left(\mathcal{C}_{0}, \mathcal{C}_{1}, h_{2}: \mathcal{C}_{2} \rightarrow \mathcal{C}_{1}\right)$. We now define a uniquely determined cartesian closed functor $f^{\prime}: \mathcal{X} \rightarrow \mathcal{C}$ making the triangle

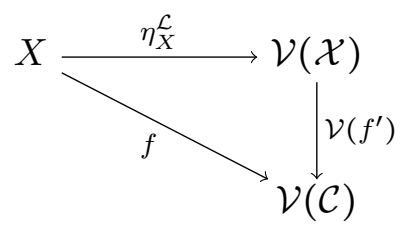

commute.

On objects, it is determined by induction: on sorts by $f_{0}$, and on type constructors by the requirement that $f^{\prime}$ be cartesian closed. On morphisms, it is similarly determined by $f_{1}$ and $f^{\prime}$ being cartesian closed. On 2-cells, define $f^{\prime}$ to be $f_{2}: X_{2}(A \vdash M, N: B) \rightarrow$ $\mathcal{C}\left(f^{\prime}(A), f^{\prime}(B)\right)\left(f^{\prime}(M), f^{\prime}(N)\right)$, which is also the only possible choice from $f$.

This indeed makes the above triangle commute, because any $r \in X_{2}(G \vdash M, N: A)$ is first mapped to $h\left(r\left\langle\pi_{1} x, \ldots, \pi_{n} x\right\rangle\right) \in X_{2}\left(\Pi G \vdash M^{\prime}, N^{\prime}: A\right)$, and then to

$$
h\left(r\left\langle\pi_{1} x, \ldots, \pi_{n} x\right\rangle\right)
$$

in $\mathcal{C}$, which, because $f$ is a morphism of $\mathcal{L}$-algebras, is equal to

$$
h_{2}\left(\left(f_{2}(r)\right)\left\langle\left\langle\pi_{1} x, \ldots, \pi_{n} x\right\rangle\right),\right.
$$

i.e., to $f_{2}(r)$.

It thus remains to show that $f^{\prime}$ is cartesian closed, which follows by $f$ being a morphism of $\mathcal{L}$-algebras. For example, to show that binary pairings of reductions are preserved, consider $r \in X_{2}\left(C \vdash M_{1}, M_{2}: A\right)$ and $s \in X_{2}\left(C \vdash N_{1}, N_{2}: B\right)$. Their product in $\mathcal{F}(X)$ is obtained by considering the atomic reductions $x: C \vdash r\left\langle\langle x\rangle: M_{1} \rightarrow M_{2}: A\right.$ and $x: C \vdash s\left\langle\langle x\rangle: N_{1} \rightarrow N_{2}: B\right.$ and taking $h\left(r\langle\langle x\rangle\rangle, s\langle\langle x\rangle)\right.$, which is mapped by $f_{2}$ to $f_{2}(h(r\langle\langle x\rangle, s\langle\langle x\rangle))$. But, because $f$ is a morphism of $\mathcal{L}$-algebras, this is the same as $h_{2}\left(\left(f_{2}(r)\right)\langle\langle x\rangle\rangle,\left(f_{2}(s)\right)\langle\langle x\rangle)\right.$, which is by definition (i.e., Figure 2) the pairing $\left(f_{2}(r), f_{2}(s)\right)$ in $\mathcal{C}$.

Acknowledgements. Thanks to Nicolas Tabareau for useful feedback, and to Aurore Alcolei for reviving the subect after a few years. 


\section{REFERENCES}

[1] H. J. Sander Bruggink. Equivalence of reductions in higher-order rewriting. PhD thesis, Utrecht University, 2008.

[2] Paolo Capriotti. Concurrent semantics with variable binding. Master's thesis, University of Pisa, 2009.

[3] Andrea Corradini, Fabio Gadducci, and Ugo Montanari. Relating two categorial models of term rewriting. In Jieh Hsiang, editor, RTA, volume 914 of Lecture Notes in Computer Science, pages 225-240. Springer, 1995.

[4] R. L. Crole. Categories for Types. Cambridge Mathematical Textbooks. Cambridge University Press, 1993. xvii+335 pages, ISBN 0521450926HB, 0521457017PB.

[5] Thierry Despeyroux and André Hirschowitz. Principles for functional abstract syntax. Draft, 1995.

[6] Uffe Engberg and Mogens Nielsen. A calculus of communicating systems with label passing. Technical Report PB-208, Aarhus University, 1986.

[7] Marcelo P. Fiore. Second-order and dependently-sorted abstract syntax. In LICS '08, pages 57-68. IEEE Computer Society, 2008.

[8] Barney P. Hilken. Towards a proof theory of rewriting: The simply typed $2 \lambda$-calculus. Theor. Comput. Sci., 170(1-2):407-444, 1996.

[9] André Hirschowitz and Marco Maggesi. Modules over monads and linearity. In Daniel Leivant and Ruy J. G. B. de Queiroz, editors, WoLLIC, volume 4576 of Lecture Notes in Computer Science, pages 218-237. Springer, 2007.

[10] Yoshiki Kinoshita, John Power, and Makoto Takeyama. Sketches. Journal of Pure and Applied Algebra, 143(1-3), 1999.

[11] Jan W. Klop. Combinatory Reduction Systems. PhD thesis, CWI, Amsterdam, 1980.

[12] Joachim Lambek and Philip J. Scott. Introduction to Higher-Order Categorical Logic. Cambridge University Press, 1986.

[13] Robin Milner. A Calculus of Communicating Systems, volume 92 of LNCS. Springer, 1980.

[14] Robin Milner, Joachim Parrow, and David Walker. A calculus of mobile processes, I/II. Information and Computation, 100(1):1-77, 1992.

[15] Tobias Nipkow. Higher-order critical pairs. In LICS '91, pages 342-349. IEEE Computer Society, 1991.

[16] TeReSe, editor. Term Rewriting Systems. Number 55 in Cambridge Tracts in Theoretical Computer Science. Cambridge University Press, 2003.

[17] Vincent van Oostrom and Femke van Raamsdonk. Comparing combinatory reduction systems and higher-order rewrite systems. In Jan Heering, Karl Meinke, Bernhard Möller, and Tobias Nipkow, editors, HOA, volume 816 of Lecture Notes in Computer Science, pages 276-304. Springer, 1993.

[18] Charles Wells. A generalization of the concept of sketch. Theor. Comput. Sci., 70(1):159-178, 1990.

[19] David A. Wolfram. The Clausal Theory of Types. Number 21 in Cambridge Tracts in Theoretical Computer Science. Cambridge University Press, 1993.

[20] Julianna Zsidó. Typed Abstract Syntax. PhD thesis, Université de Nice-Sophia Antipolis, 2010. 
Appendix A. Equations on Reductions

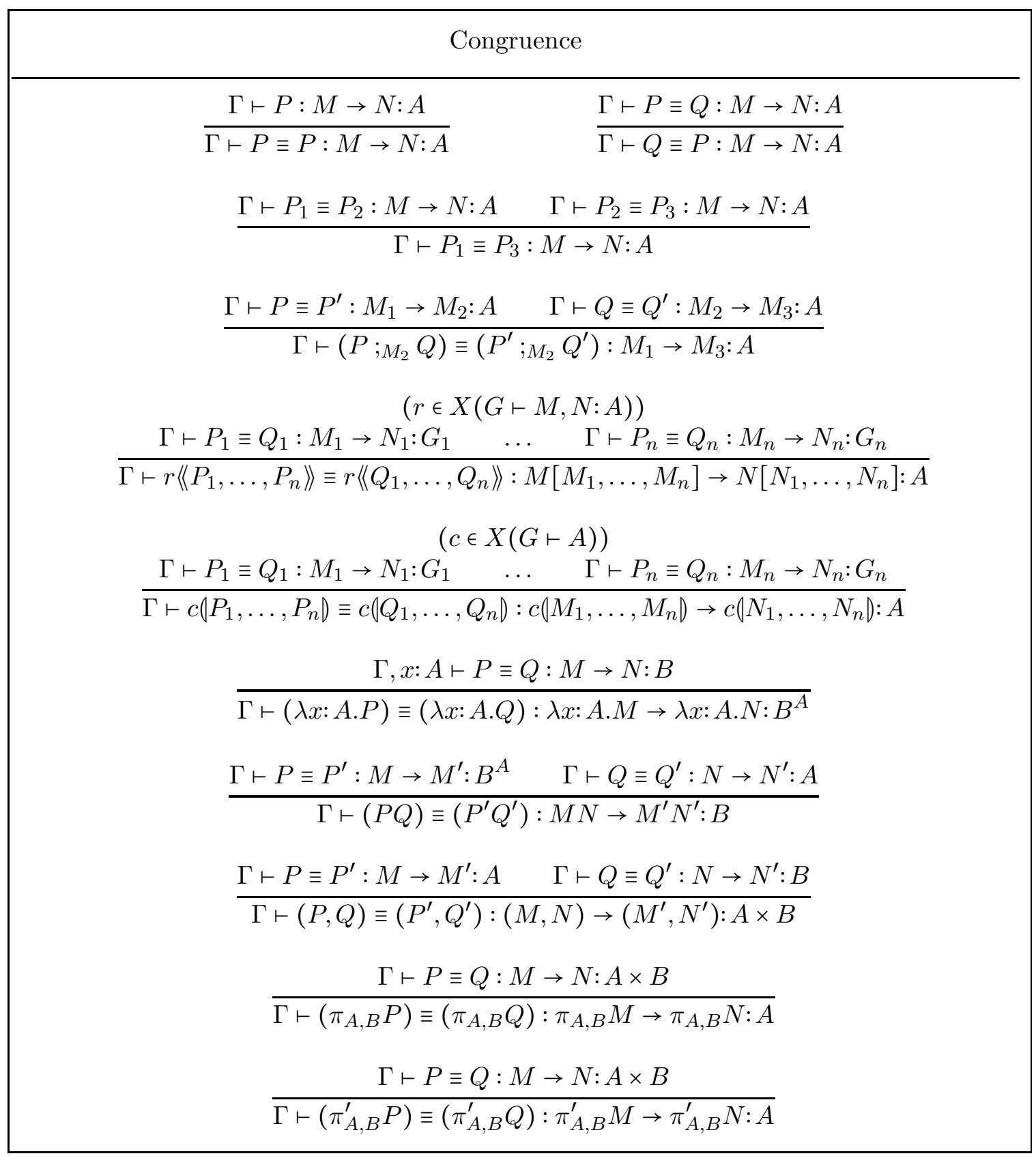

Figure 3: Equations on reductions (Congruence) 


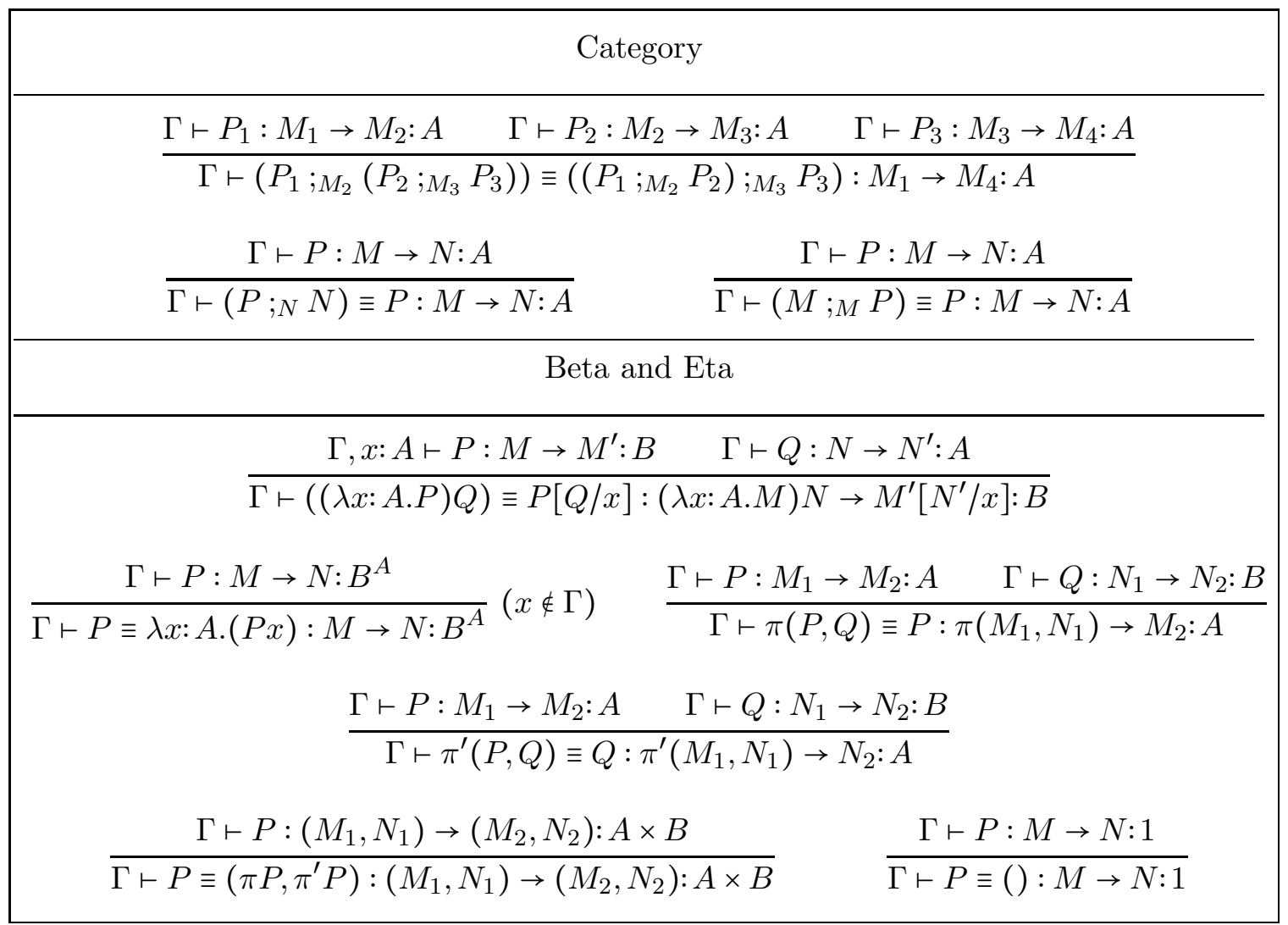

Figure 4: Equations on reductions (Category and Beta-Eta) 


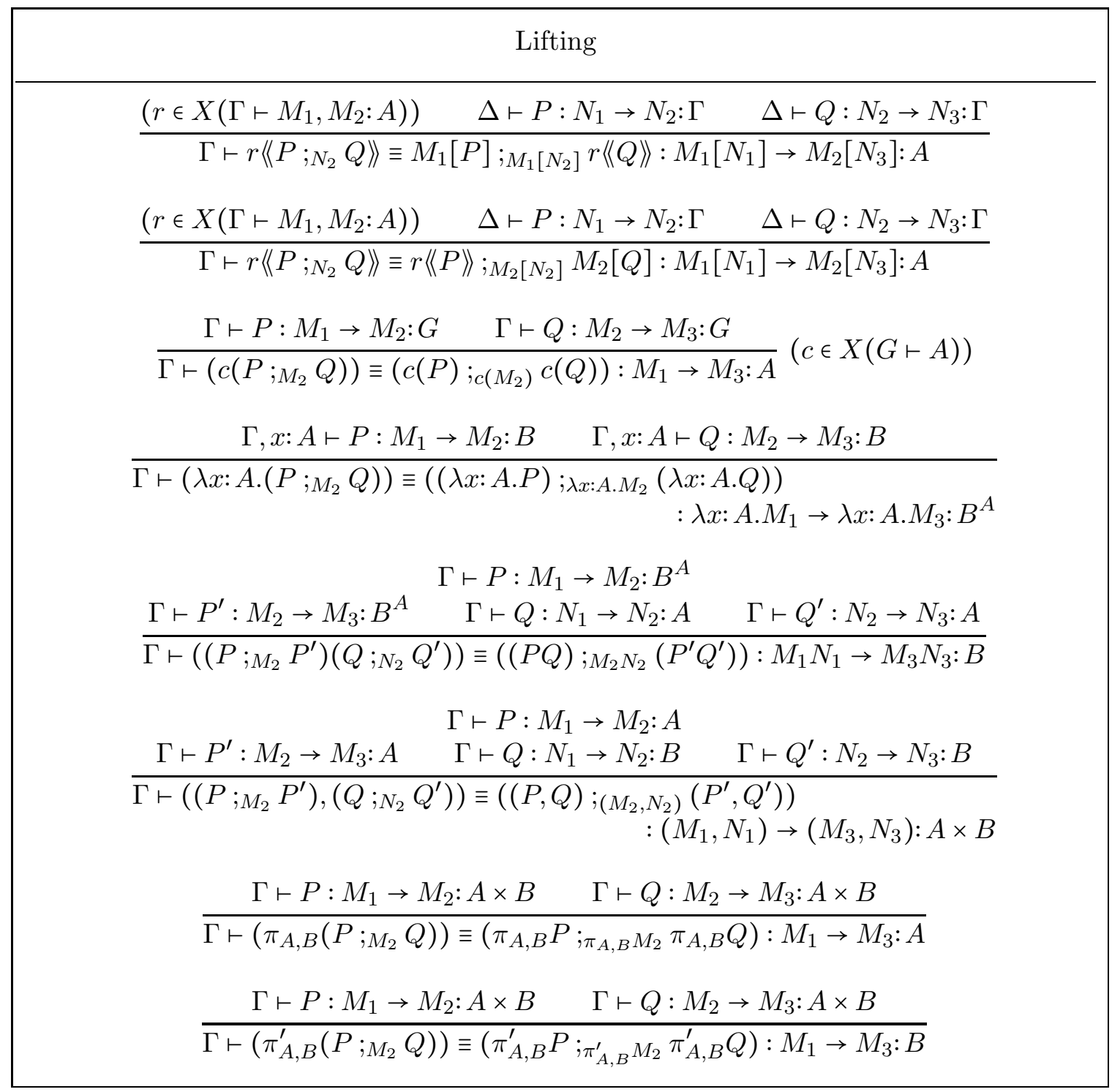

Figure 5: Equations on reductions (Lifting) 